\title{
Oral Iron Therapy-Induced Neutropenia in Patient with Iron Deficiency Anemia
}

\author{
Mohammad N. Kloub ${ }^{a}$ Mohamed A. Yassin ${ }^{b}$ \\ aDepartment of Internal Medicine, Hamad Medical Corporation, Doha, Qatar; ${ }^{b}$ Department \\ of Medical Oncology, National Center for Cancer Care and Research, Doha, Qatar
}

\section{Keywords}

Iron deficiency anemia $\cdot$ Leukopenia $\cdot$ Neutropenia $\cdot$ Lymphopenia $\cdot$ Anemia $\cdot$ Iron

\begin{abstract}
Iron deficiency anemia is common and worldwide distributed, particularly among females; however, it can also occur among males. Iron deficiency anemia is commonly associated with thrombocytosis; little is known about the effect of iron therapy (oral or intravenous) on other hematological parameters. We report a 29-year-old male patient with iron deficiency anemia, who received oral iron replacement therapy and developed neutropenia which recovered spontaneously 1 month later.

\section{Introduction}

"Iron deficiency is defined as a condition in which there are no mobilizable iron stores and in which signs of a compromised supply of iron to tissues, including the erythron, are noted. The more severe stages of iron deficiency are associated with anaemia. When irondeficient erythropoiesis occurs, haemoglobin concentrations are reduced to below-optimal levels. When individual haemoglobin levels are below two standard deviations (-2SD) of the distribution mean for haemoglobin in an otherwise normal population of the same gender and age who are living at the same altitude, iron deficiency anaemia is considered to be present" [1].

Iron deficiency anemia (IDA) can be treated by treatment of the underlying cause and the deficient iron can be corrected by iron replacement therapy, which can be in the form of oral or intravenous treatment; the effect of oral and intravenous iron on hematological parameters like leukocytes has not been well studied. And since little is known about oral ironinduced leukopenia which is a rare entity, we would like to shed light on this important aspect. 


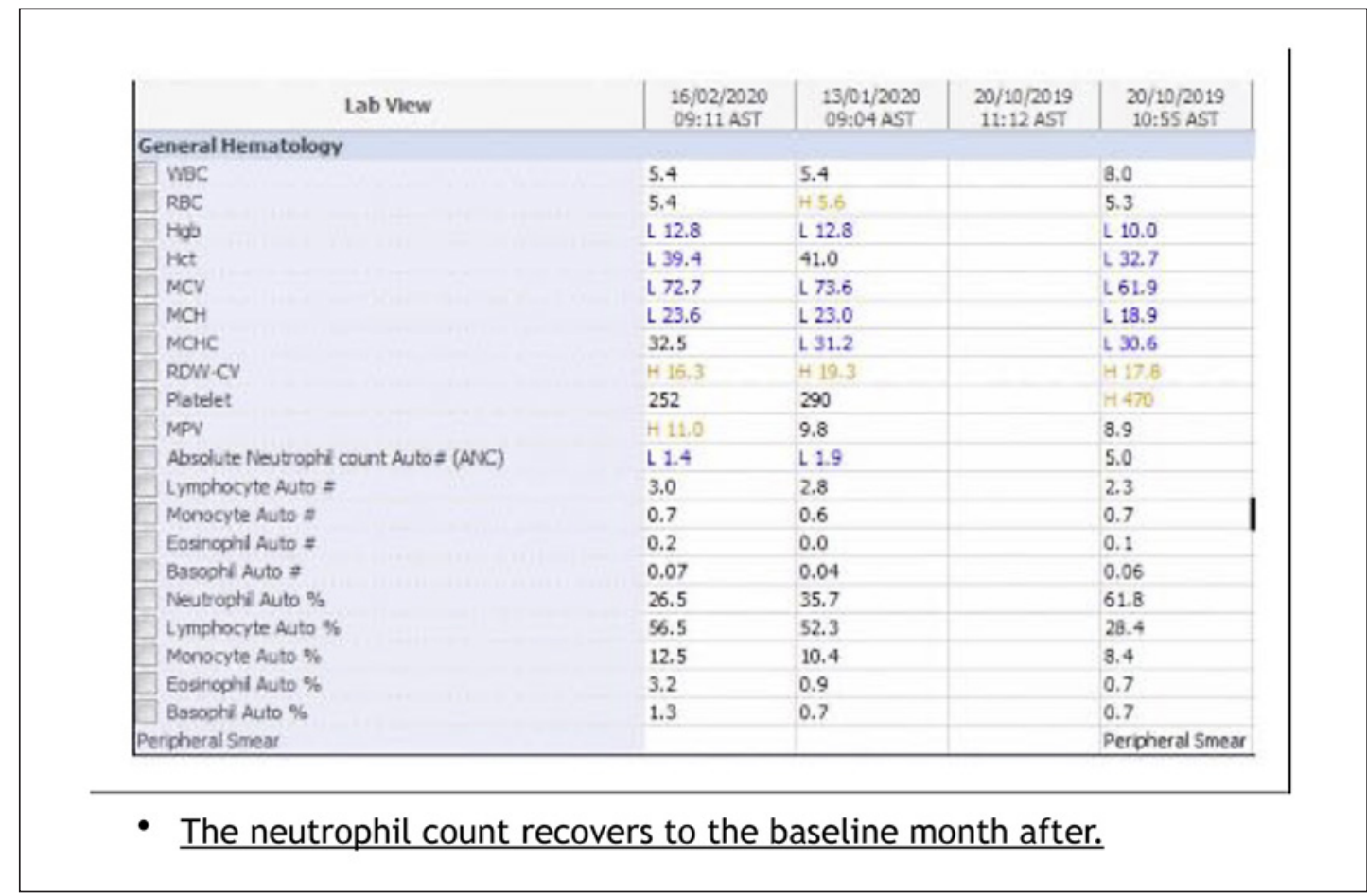

Fig. 1. The neutrophil count recovers to the baseline month afterwards.

\section{Case Report}

29-year-old male patient, known to have thalassemia trait, status post-sleeve gastrectomy in 2013, complicated by IDA.

Follow-up CBC showed microcytic anemia with $\mathrm{Hb} 10 \mathrm{~g} / \mathrm{dL}$ (normal range13-17), MCV $61.9 \mathrm{fL}$ (normal range 83-101), platelets 470,000 (normal range 150,000-400,000), iron level $3 \mu \mathrm{mol} / \mathrm{L}$ (normal range 6-35), TIBC $108 \mu \mathrm{mol} / \mathrm{L}$ (normal range 45-80), transferrin 4.3 $\mathrm{g} / \mathrm{L}$ (normal range 2.0-3.6), and iron saturation 3\% (normal range 15-45\%).

He was seen at the hematology clinic and labeled as a case of IDA secondary to malabsorption post-gastric surgery, and started on oral iron replacement (ferrous fumarate $200 \mathrm{mg}$ bid daily).

Repeated CBC after 2 months showed $\mathrm{Hb} 12.80 \mathrm{~g} / \mathrm{dL}$ (normal 13-17), MCV $72.7 \mathrm{fL}$ (83-101), and platelets 252 (normal 150,000-400,000).

However due to ferrous fumarate, the patient developed neutropenia, as illustrated in Figure 1.

\section{Discussion/Conclusion}

Anemia is a global public health problem affecting both developing and developed countries with major consequences for human health as well as social and economic development. It occurs at all stages of the life cycle [2].

IDA has a direct impact on quality of life in different aspects which include male fertility; the correction of IDA with IVI is associated with significant enhancement of sperm parameters and increased concentrations of serum LH, FSH, and T. These effects on spermato- 
genesis are achieved by an unknown mechanism and suggest several pathways that need further human and/or experimental studies [3].

Iron replacement therapy decreases HbA1c in both diabetic and non-diabetic individuals. This implies that the iron states must be considered during the interpretation of $\mathrm{HbA1c}$ concentrations in diabetic or non-diabetic patients. Early diagnosis and treatment of ID in diabetic patients can improve their glycemic control and may prevent or delay complications [4].

Leukopenia is an abnormal reduction in the number of circulating white blood cells, namely granulocytes. Neutropenia is a more specific term referring to the abnormal reduction in the number of circulating neutrophils [5], which is the focus of our case report here. Neutropenia occurs when there is a reduction in the number of produced neutrophils or when there is increased destruction, or both. Infection, drugs, malignancy, megaloblasts, hypersplenism, and immune neutropenia are known causes of leukopenia [6]. The primary threat when dealing with neutropenia lies in the increased risk and severity of infection [7].

Lim et al. [8]reported in one abstract a high incidence of leukopenia in patients with IDA. The severity of leukopenia correlates with the decrease of hemoglobin in a dose-dependent manner. Among the WBC subsets, lymphocyte count was significantly affected. According to our knowledge, this is the first case documenting neutropenia after oral iron therapy.

\section{Conclusion}

Neutropenia can occur after oral replacement therapy and it should be looked for; however, if it occurs, recovery is spontaneous.

\section{Acknowledgment}

The authors would like to acknowledge the Internal Medicine and Hematology Department at Hamad Medical Corporation for supporting this publication.

\section{Statement of Ethics}

Written informed consent was obtained from the patient to allow the publication of information including images. Case approved by HMC Medical Research Center.

\section{Disclosure Statement}

The authors report no conflicts of interest in this work.

\section{Funding Sources}

Qatar National Library.

\section{Authors Contribution}

Both authors contributed equally in writing and editing. 


\section{References}

1 Kloub MN. Iron Deficiency Anaemia Assessment. Prevention and Control: a guide for programme managers. World Health Organization; 2001.

2 Soliman AT, De Sanctis V, Yassin M, Wagdy M, Soliman N. Chronic anemia and thyroid function. Acta Biomed. 2017;88(1):119.

3 Soliman A, Yassin M, De Sanctis V. Intravenous iron replacement therapy in eugonadal males with iron-deficiency anemia: effects on pituitary gonadal axis and sperm parameters; a pilot study. Indian J Endocrinol Metab. 2014 May;18(3):310, 6.

4 Soliman AT, De Sanctis V, Yassin M, Soliman N. Iron deficiency anemia and glucose metabolism. Acta Biomed. 2017;88(1):112-8.

5 Donadieu J, Fenneteau O, Beaupain B, Mahlaoui N, Chantelot CB. Congenital neutropenia: diagnosis, molecular bases and patient management. Orphanet J Rare Dis. 2011;6:26-54.

6 Schneeweiss S, Hasford J, Göttler M, Hoffmann A, Riethling AK, Avorn J. Admissions caused by adverse drug events to internal medicine and emergency departments in hospitals: a longitudinal population-based study. Eur J Clin Pharmacol. 2002;58(4):285-91.

7 Lee GR, Wintrobe MM, Foerster J, Lukens J, Paraskevas F, Parodi JC, et al. Wintrobe's hematology. 10th ed. Baltimore, MD: William and Wilkins; 1999. p. 1836-88.

8 Lim Y, Lee EY, Choi IS, Kim T-Y, Yoon S-S, Kim KH. Leukopenia in patients with iron deficiency anemia. Blood. 2011;118(21):5279. 